\title{
Reversibility of gastric mucosal lesions induced by sodium phosphate tablets and characterized by probe-based confocal laser endomicroscopy
}

Authors

Institutions
Emmanuel Coron ${ }^{1,2,3}$, Marie Dewitte ${ }^{1,2,3}$, Philippe Aubert ${ }^{1,2,3}$, Nicolas Musquer ${ }^{1}$, Michel Neunlist ${ }^{1,2,3}$, Stanislas Bruley des Varannes $1,2,3$

${ }^{1}$ Hôtel Dieu, Centre Hospitalier Universitaire, Institut des Maladies de l'Appareil Digestif, Nantes, France 2 INSERM U 913, Nantes, France

3 Université de Nantes, Faculté de Médecine, Nantes, France submitted

14. February 2014 accepted after revision: 15. July 2014

\section{Bibliography}

Dol http://dx.doi.org/

10.1055/s-0034-1377934

Published online: 30.10 .2014

Endoscopy International Open

2015; 03: E69-E75

(c) Georg Thieme Verlag KG

Stuttgart · New York

E-ISSN 2196-9736

\section{Corresponding author}

\section{Emmanuel Coron, MD, PhD}

Institut des Maladies de

l'Appareil Digestif -

INSERM U 913

CHU Hôtel Dieu

44093 Nantes Cedex

France

Fax: +33 (0)2 40083154

emmanuel.coron@chu-nantes.fr
Background and study aims: Adequate bowel preparation is key for the optimal quality of colonoscopy. The sodium phosphate laxatives used for preparation may induce gastric injuries. However, in vivo studies monitoring the effects of sodium phosphate on the gastric mucosa are currently lacking. We aimed to characterize the effects of sodium phosphate tablets (Colokit ${ }^{\circledR}$; Mayoly Spindler, Chatou, France) on the gastric mucosa in a large-animal model.

Methods: Fourteen anesthetized pigs were used for this study. Fundic mucosal sites were analyzed at $1.5,24$, and 72 hours after the endoscopically guided application of sodium phosphate tablets (NaPT) and placebo tablets (PlaT) and were compared with unexposed sites. Different mucosal parameters were assessed with white light endoscopy, probe-based confocal laser endomicroscopy (pCLE), histology, and ex vivo permeability measurements.

\section{Introduction}

\section{$\nabla$}

Several parameters of quality have been defined for optimal colonoscopy, such as cecal intubation, adverse event rates, duration of withdrawal, and bowel preparation [1-5]. High-quality bowel preparation is noted to be of primary importance for an effective colonoscopic examination.

Among bowel preparation agents, osmotic laxatives (i.e., mainly water-soluble polymers that are neither digested nor absorbed in the small intestine) have been widely used for more than three decades [6]. The osmotic action of these agents increases the colonic fluid volume by maintaining water in the colonic lumen, thereby distending the bowel and indirectly increasing bowel peristalsis.

More recently, osmotic laxatives containing sodium phosphate have been proposed, in order to decrease the volume of fluid intake and improve patient acceptability [7-10]. However, recent
Results: At 90 minutes after the application of $\mathrm{NaPT}$, significant increases in epithelial irregularity and crypt pit intensity were observed with pCLE. These microscopic lesions persisted at 24 hours but were resolved at 72 hours. In addition, white light endoscopy revealed local exanthema in $57 \%$ of the animals at 1.5 hours after NaPT application. Such lesions were observed in $22 \%$ of the pigs at 24 hours and disappeared at 72 hours after application. After 1.5 hours, PlaT induced a slight but significant increase in epithelial irregularity, as well as architectural scores that were significantly lower than the ones induced by NaPT and that disappeared after 72 hours.

Conclusions: The direct and prolonged gastric application of NaPT in pigs can induce acute superficial macroscopic and microscopic injuries that are reversible within 72 hours.

studies have reported that these agents, in solution or in a tablet formulation, may induce aphthous or erosive injuries of the stomach and colon in some patients [11-15]. In addition, after the introduction of sodium phosphate tablets (NaPT) in France, several individual case safety reports of gastritis or stomach ulcerations were registered. To date, no risk factors, such as preexisting lesions, history, co-morbidities, and concomitant drug use, have been identified. Although these lesions are frequently asymptomatic and can be viewed as "false-positive" findings, it is important to explain their pathophysiology and evolution more clearly. For instance, although the specific mechanisms inducing such lesions remain unknown, it has been suggested that an increase in the production of free radicals may be involved [16]. However, defining these osmotic laxativeinduced lesions in humans remains difficult. Therefore, it was thought that an experimental model would be helpful by allowing time course 


\begin{tabular}{|c|c|c|c|c|c|}
\hline & $\stackrel{\mathrm{H} 0}{\llcorner}$ & $\mathrm{H} 1.5$ & $\mathrm{H}_{2} 4$ & $\mathrm{H} 72$ & \\
\hline CTRL-NaPT & & & & & Study 1 \\
\hline CTRL-PLaT-NaPT & \multicolumn{3}{|c|}{$P_{6}-P_{10}$} & & Study 2 \\
\hline CTRL-PLaT-NaPT & \multicolumn{3}{|c|}{$P_{11}-P_{14}$} & & Study 3 \\
\hline $\begin{array}{l}\text { Macroscopic } \\
\text { view }\end{array}$ & + & + & + & + & \\
\hline pCLE recording & + & + & + & + & \\
\hline $\begin{array}{l}\text { Paracellular } \\
\text { permeability }\end{array}$ & + & + & + & + & \\
\hline
\end{tabular}

Fig. 1 Study design. In study $1(\mathrm{n}=5)$, analysis was performed on sodium phosphate tablet (NaPT) sites at 1.5 hours after the application of NaPT tablets and on unexposed mucosa, referred to as control (CTRL) sites. In studies 2 and $3(n=9)$, analysis was performed on NaPT, PLaT, and CTRL sites at 1.5, 24, and 72 hours after the application of NaPT and placebo tablets (PLaT). At each considered time point ( $0,1.5,24$, and 72 hours), the endoscopic aspect was reported, and probe-based confocal laser endomicroscopy ( $\mathrm{PCLE}$ ) was carried out for 1 minute at each site.

studies to be done. Such an experimental model should fulfill several criteria, enabling (1) prolonged and stable exposure to laxative or control substances, (2) in vivo and possibly repeated endoscopic examinations, and (3) histologic and functional analysis of the exposed mucosa.

In an attempt to reproduce the gastric lesions observed in humans, we developed a pig model to determine the effects of the prolonged and direct application of NaPT on the gastric mucosa by using a combination of endoscopic, probe-based confocal laser endomicroscopy (pCLE) and histologic and functional methods.

\section{Materials and Methods}

$\nabla$

\section{Animals and tablets}

A total of 14 pigs (weighing between 30 and $40 \mathrm{~kg}$ ) were used in this study. Experiments were done in a dedicated surgical facility of the Laboratoire des Grands Animaux, INSERM U 643, in accordance with French Veterinary Regulations and Ethics Committee standards (agreement E.44010). During all procedures, the animals were under general anesthesia, induced with $5 \%$ isoflurane and $60 \%$ nitrous oxide and subsequently maintained with $2 \%$ isoflurane.

Each NaPT (Colokit ${ }^{\circledR}$; Mayoly Spindler, Chatou, France) weighed $1500 \mathrm{mg}$ and was composed of $1102 \mathrm{mg}$ of monobasic sodium phosphate monohydrate and $398 \mathrm{mg}$ of dibasic anhydrous sodium phosphate. Each placebo tablet (PlaT) weighed $1704 \mathrm{mg}$ and was composed of $170.4 \mathrm{mg}$ of polyethylene glycol, $8.52 \mathrm{mg}$ of magnesium stearate, $1354.68 \mathrm{mg}$ of lactose (Tablettose ${ }^{\circledR}$; Meg- gle, Wasserburg, Germany), and $170.4 \mathrm{mg}$ of microcrystalline cellulose (Avicel ${ }^{\circledR}$; FMC BioPolymer, Philadelphia, Pennsylvania, USA).

\section{Study design}

The study design is summarized in Fig. 1. An initial study (study 1 ) was conducted with five pigs to determine the feasibility of the procedure and to characterize early lesions observed after NaPT had been applied for 1.5 hours. Two consecutives studies were then performed to determine the time course of the lesional aspects at 1.5 hours, 24 hours (study $2, \mathrm{n}=5$ ), and 72 hours (study 3, n=4) after the application of NaPT and PlaT. In order to consider potential mechanical effects of the tablets, a normal mucosal area, hereafter referred as the control site, was also analyzed. Thus, three different sites of analysis were considered: control, PlaT, and NaPT.

At each considered time $(0,1.5,24$, and 72 hours $)$, both the macroscopic and microscopic (pCLE) aspects of each site were analyzed in vivo during upper gastrointestinal endoscopy. At the end of the study, an endoscopic mucosal resection (EMR) was performed on each site, and specimens were sent for further ex vivo morphologic and functional analysis.

\section{Endoscopic procedure}

During all procedures, performed by a single experienced endoscopist (E.C.), the animals were in prone position and under general anesthesia. The endoscopic appearance of the gastric mucosa was assessed meticulously with a standard resolution endoscope (EG450; Fujifilm, Tokyo, Japan). First, particular attention was paid to washing the gastric cavity with syringes of water. The best area on which to apply both tablets (PlaT and NaPT) was then defined as a stable area in the fundus with no visible contractions. One NaPT tablet was captured with an endoscopic snare, stabilized within a cap placed at the distal tip of the endoscope, and placed into the stomach at the level of the fundus. The same procedure was immediately repeated with a PlaT, which was placed 2 to $3 \mathrm{~cm}$ to the right of the previous tablet. Finally, one endoscopic clip was positioned between the two tablets in order to allow re-identification of these areas at 24 and 72 hours ( $\bullet$ Fig.2). The macroscopic aspect of each site of analysis was described and graded as follows: grade 0 , no lesion; grade 1 , mild lesion defined by the presence of exanthema; grade 2 , moderate lesion defined by the presence of aphthous ulcer and/or black clots; grade 3, severe lesion defined by the presence of ulcer and/or active bleeding.
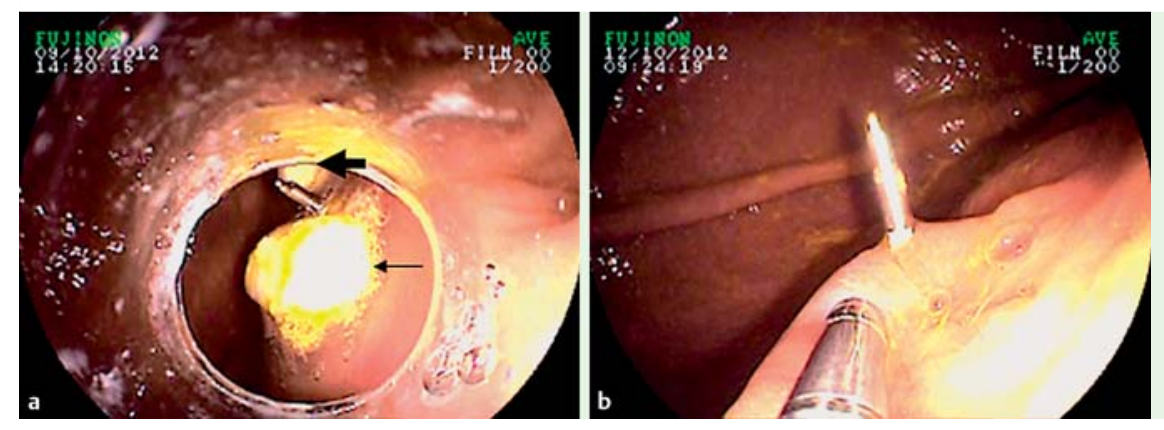

Fig. 2 Endoscopic views. a The drug tablets - that is, the sodium phosphate tablet (thin arrow) and the placebo tablet (thick arrow) - a few minutes after endoscopic placement on the fundic mucosa of pigs. b The same sites at 24 hours. The endoscopic clip was initially positioned between the two tablets to allow re-identification of the sites after complete dissolution of the tablets. 


\section{Probe-based confocal laser endomicroscopy} examination

The PCLE procedure was performed by a single experienced endoscopist (E.C.), as follows. The confocal miniprobe (ColoFlexUHD, Cellvizio; Mauna Kea Technologies, Paris, France) was gently positioned onto the gastric mucosa in contact with either the drug tablet or the placebo tablet, or onto the control site. A volume of $5 \mathrm{~mL}$ of $10 \%$ fluorescein sodium (Novartis Pharma, France) was injected intravenously as a contrast agent. The pCLE video sequences were recorded from the time of injection to 5 minutes after injection for further "off-line" analysis.

Following the procedure, each video clip was reviewed by one endoscopist (M.P.), who was blinded to the procedure. The six best images from each pCLE video sequence were extracted and transferred to an external drive in PNG (portable network graphics) format to allow further semiquantitative analysis. The final data set comprised 678 images from 14 pigs. The following parameters were assessed: epithelial lining irregularity, architectural disorganization, and fluorescein intensity, which was measured in both the crypt lumen and the intercryptic area. ImageJ $1.42 \mathrm{q}$ software was used to measure fluorescein intensity (National Institutes of Health, Bethesda, Maryland, USA). Epithelial irregularity and architectural disorganization were assessed with a semiquantitative score ( $\bullet$ Table $\mathbf{1}$ ) adapted from Li et al. [17]. This score was assessed independently by two procedureblinded investigators (M.D. and N.M.).

\section{Ex vivo assessment of paracellular permeability}

Fundic specimens obtained by EMR were immediately placed into Krebs solution $\left(0.187 \mathrm{~g} / \mathrm{L} \mathrm{NaH}_{2} \mathrm{PO}_{4} \cdot 2 \mathrm{H}_{2} \mathrm{O}, 6.84 \mathrm{~g} / \mathrm{L} \mathrm{NaCl}, 0.35\right.$ $\mathrm{g} / \mathrm{L} \mathrm{KCl}, 2.10 \mathrm{~g} / \mathrm{L} \mathrm{NaHCO}{ }_{3}, 1.98 \mathrm{~g} / \mathrm{L}$ glucose, $0.368 \mathrm{~g} / \mathrm{L} \mathrm{CaCl}{ }_{2} \cdot 2 \mathrm{H}_{2} \mathrm{O}$, $0.244 \mathrm{~g} / \mathrm{L} \mathrm{MgCl}_{2} \cdot 6 \mathrm{H}_{2} \mathrm{O}$ ) at $4^{\circ} \mathrm{C}$. For each site, the EMR specimen was microdissected to separate the mucosa from the submucosal layer (in the plane of the submucosal blood vessels). Specimens of isolated fundic mucosa were then mounted in dedicated Ussing chambers (Physiologic Instruments, San Diego, California, USA) with a chamber surface of $0.0314 \mathrm{~cm}^{2}$. Each chamber contained $2 \mathrm{~mL}$ of Ham's F12 Nutrient Mixture (Life Technologies, Grand Island, New York, USA) containing $0.1 \%$ fetal calf serum. The media were continuously oxygenated by a gas flow of $95 \% \mathrm{O}_{2}$ and $5 \%$ $\mathrm{CO}_{2}$ and maintained at $37^{\circ} \mathrm{C}$. After an equilibration period of 30 minutes, $200 \mu \mathrm{L}$ of apical medium was replaced with $200 \mu \mathrm{L}$ of fluorescein -5.6 sulfonic acid $(1 \mathrm{mg} / \mathrm{mL})$. Every 30 minutes, the fluorescence level of basolateral aliquots of $150 \mu \mathrm{L}$ was measured over a period of 180 minutes with a fluorometer (Thermo Scientific). Paracellular permeability was determined by calculating the slope of the changes in fluorescence intensity over time with a linear regression fit model (GraphPad Software, La Jolla, California, USA).

\section{Histologic evaluation}

For each site, a portion of the EMR specimen was fixed in $4 \%$ paraformaldehyde in phosphate-buffered saline. After tissue washing in phosphate-buffered saline, the specimen was dehydrated and embedded in paraffin. Sequential sections of mucosa ( $5 \mu \mathrm{m})$ were obtained and, after staining in hematoxylin and eosin, were analyzed with an Olympus IX50 microscope (Olympus America, Center Valley, Pennsylvania, USA). Pictures were captured with a DP71 digital camera (Olympus) connected to a computer through a frame grabber card (Cell^ ${ }^{\wedge}$ software, Olympus). To assess the mucosal morphology, we analyzed mucosal thickness, determined by measuring the distance between the surface epithelium and the underlying muscularis mucosae (only in study 1 ).

\section{Statistical analysis}

Because of the limited sample size, nonparametric tests were used for statistical analysis. Two groups were compared with either the Mann-Whitney test or the Wilcoxon test. More than two groups were compared with either the Kruskal-Wallis test or the Dunn test. Comparisons between groups, and over time, were performed with a two-way analysis of variance (ANOVA) followed by a Bonferroni test. All statistical analyses were performed with GraphPad Prism 5.00 for Windows (GraphPad Software).

\section{Results}

$\nabla$

Induction of specific, early gastric mucosal lesions with sodium phosphate tablets

All tablets were successfully applied onto the fundic mucosa under endoscopic guidance ( $\bullet$ Fig.2). At 1.5 hours, all tablets remained in position at the original site and were partially dissolved.

First, we used white light endoscopy to grade the mucosal changes induced by NaPT. All sites in all animals were macroscopically normal (grade 0 ) at the time of NaPT application. At 1.5 hours, only grade 1 lesions were observed in 8 of the 14 animals (57\%) at the NaPT sites. No grade 2 or 3 lesions were observed. No lesions were observed at control sites.

Second, we used pCLE to analyze and quantify microscopic mucosal changes in vivo. With semiquantitative score, we indicated that all sites initially had normal epithelial lining and glandular architecture (grade 0 ). At 1.5 hours, no epithelial irregularity or architectural disorganization was seen at control sites. In contrast, a significant increase in epithelial irregularity and architectural disorganization scores was noted at NaPT sites ( $\mathbf{F i g} . \mathbf{3}$ a, b). Blinded interobserver analysis showed an acceptable match between two independent investigators (kappa $=0.52$ ). Further quantitative pCLE analysis showed that changes in pit intensity over 1.5 hours were significantly greater at NaPT sites than at control sites. In contrast, changes in intercryptic intensity did not differ between control and NaPT sites during this time interval $(\bullet \mathbf{F i g} . \mathbf{3} \mathbf{c}, \mathbf{d})$.

Third, in addition to in vivo endoscopic analysis, we performed both histologic and functional analysis on EMR specimens collected at the end of this part of the study. We showed that mucosal thickness at NaPT sites was similar to that at control sites $(0.33 \pm$ $0.05 \mathrm{~mm}$ vs. $0.27 \pm 0.04 \mathrm{~mm} ; \mathrm{n}=5 ; P>0.05)$. Consistently, fundic

\begin{tabular}{|llll|}
\hline $\begin{array}{l}\text { Surface epithelium } \\
\text { architecture }\end{array}$ & Regular thickness & $\mathbf{1}$ (moderate) & $\mathbf{2}$ (severe) \\
\hline Crypts architecture & $\begin{array}{l}\text { Regular arrangement } \\
\text { and size of crypts }\end{array}$ & $\begin{array}{l}\text { Irregular arrangement of } \\
\text { crypts, enlarged } \\
\text { spaces between crypts }\end{array}$ & $\begin{array}{l}\text { Destroyed } \\
\text { epithelium }\end{array}$ \\
\hline
\end{tabular}

Table 1 Classification of endomicroscopic lesions. 

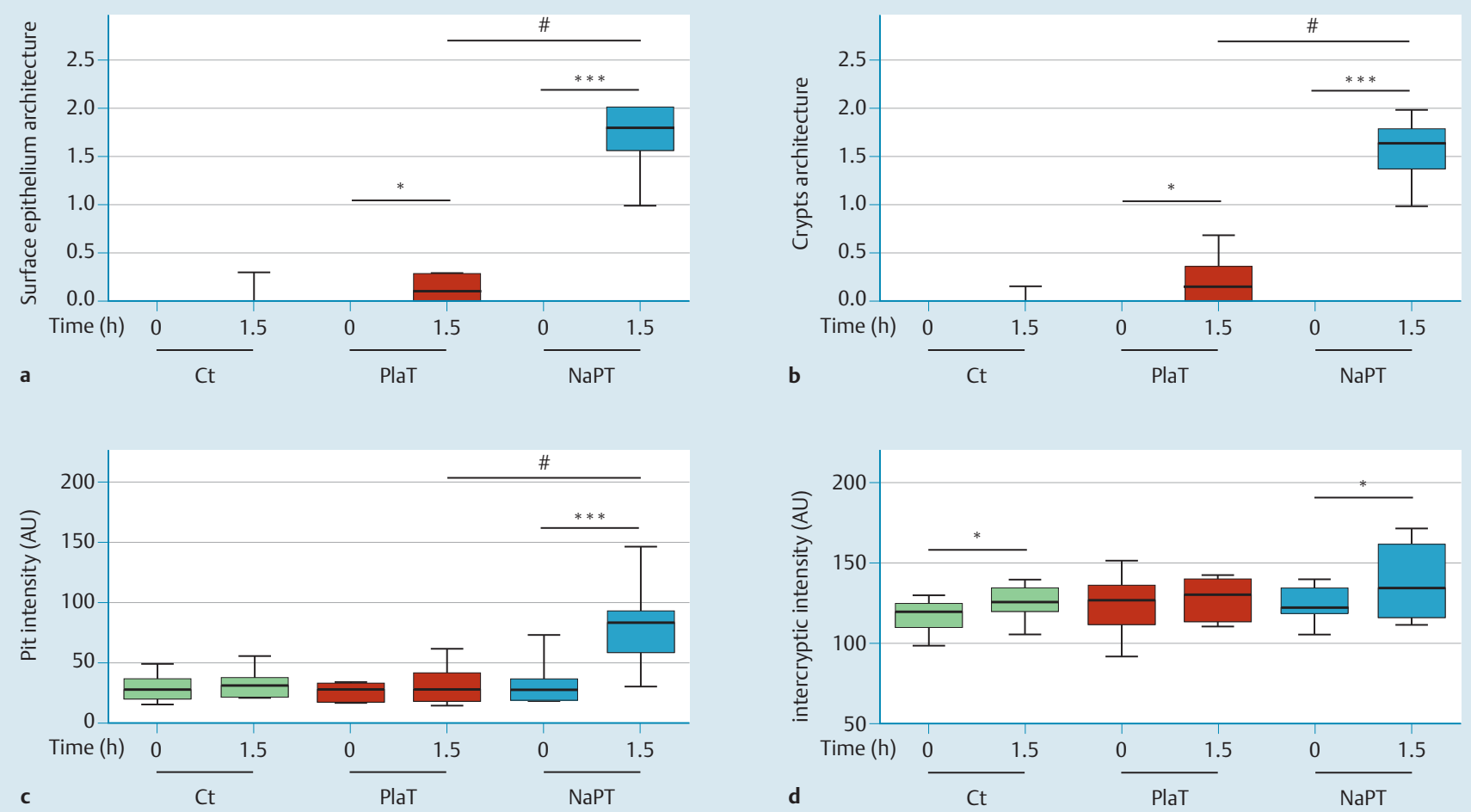

Fig. 3 Short-term effects of sodium phosphate tablets (NaPT) 1.5 hours after application onto the fundic mucosa of pigs, assessed with probe-based confocal laser endomicroscopy (studies 2 and 3 ) and graded. a Semiquantitative lesion score of surface epithelium architecture. b Semiquantitative lesion score of crypts architecture. c Variations of fluorescein intensity in the crypt lumen $[(\mathrm{H} 1.5-\mathrm{H} 0) \times 100 / \mathrm{HO}]$. d Variations of intercryptic intensity. Wilcoxon signed-rank test: ${ }^{*} P \leq 0.05,{ }^{* * *} P<0.0001$; Mann-Whitney test: ${ }^{\#} P<0.0025$. PlaT, placebo tablet; $C$ t, control; $\mathrm{AU}$, arbitrary units.

paracellular permeability was not changed at NaPT sites in comparison with control sites $(0.28 \pm 0.04$ vs. $0.33 \pm 0.05 ; n=5 ; P>$ $0.05)$. Furthermore, there was no correlation between paracellular permeability and pit or crypt intensity.

Finally, in order to consider potential mechanical effects of NaPT on mucosal lesions, we performed a comparative study with PlaT. Initially, all sites were macroscopically normal (grade 0) and remained normal 1.5 hours after application of the PlaT $(n=9)$. PlaT induced a slight but significant increase in epithelial irregularity and architectural scores ( $\bullet$ Fig. 3 a,b). However, epithelial irregularity and architectural disorganization scores were significantly higher at NaPT than at PlaT sites ( Fig. 3 a, b). PlaT did not induce a change in pit intensity at 1.5 hours after application, in contrast to NaPT ( Fig.3c). Similarly, PlaT did not induce a change in intercryptic intensity at 1.5 hours after application, in contrast to NaPT ( $\bullet$ Fig.3d).

\section{Evolution of gastric mucosal lesions induced by sodium phosphate tablets}

This part of the study attempted to monitor the evolution of the mucosal lesions induced by NaPT at 24 and 72 hours after tablet application ( Fig.4). At 24 and 72 hours, with white light endoscopy, all application sites could be easily re-identified by the presence of the endoscopic clips. There were no residual tablets at these sites.

First, macroscopic examination 24 hours after NaPT application revealed that 2 of 9 pigs had grade 1 lesions (22\%), 1 had grade 2 lesions (11\%), and the remaining 6 had no lesion (67\%). In 2 of 9 pigs ( $22 \%$ ), the PlaT site showed grade 1 lesions, and the remaining pigs had no lesion at the PlaT site. Finally, no lesion was visible at the control site in any pig. At 72 hours, no lesion was identifiable in any animal for any conditions (NaPT, PlaT, and control). Second, pCLE at PlaT and control sites showed that crypt pit intensity and intercryptic intensity remained unchanged over the different time points studied ( $\bullet$ Fig. 5 ). In contrast, at NaPT sites, the increased semiquantitative scores and crypt pit intensity observed at 1.5 hours remained unchanged at 24 hours. However, at 72 hours, the semiquantitative scores and crypt pit intensity were significantly reduced in comparison with the values at 24 hours and were similar to those measured before the application of NaPT ( $\bullet$ Figs.4,5).

Finally, we performed functional analysis on EMR specimens collected at 24 and 48 hours after tablet application. Fundic paracellular permeabilities were similar at each site and at all time points $(0.19 \pm 0.07$ vs. $0.22 \pm 0.07$ vs. $0.18 \pm 0.07$ and $0.10 \pm 0.02$ vs. $0.09 \pm 0.04$ vs. $0.10 \pm 0.03$, respectively, for NaPT, PlaT and Ct sites at 24 and 72 hours, respectively).

\section{Discussion}

$\nabla$

The present study demonstrated that a 90 -minute application of NaPT onto fundic mucosa induces endomicroscopically detectable changes in the epithelial lining. These changes persisted for 24 hours after NaPT application but were resolved at 72 hours. In addition, standard video endoscopy revealed minor macroscopic lesions, such as local exanthema or superficial lesions, at the site of NaPT application in a subgroup of animals. These lesions disappeared by 72 hours after application.

Few studies have specifically determined the gastrotoxic effects of orally administered drugs, and this characterization remains 

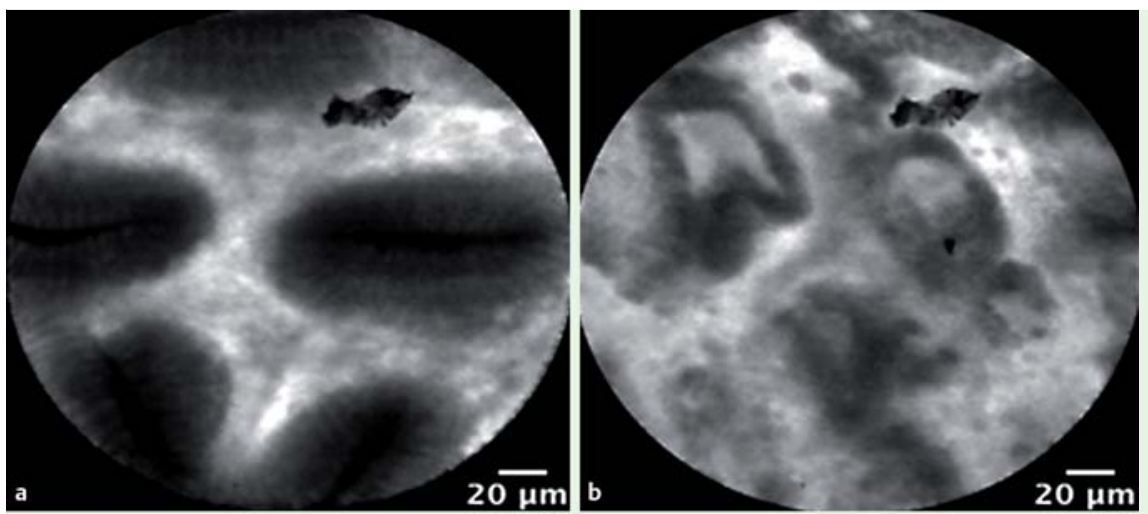

Fig. 4 Observations made in pigs with probebased confocal laser endomicroscopy at the sodium phosphate tablet site. a Normal appearance immediately before tablet application. b, c Severe epithelial irregularity and architectural disorganization at 1.5 and 24 hours, respectively. $\mathbf{d}$ Partial healing of these mucosal alterations at 72 hours.
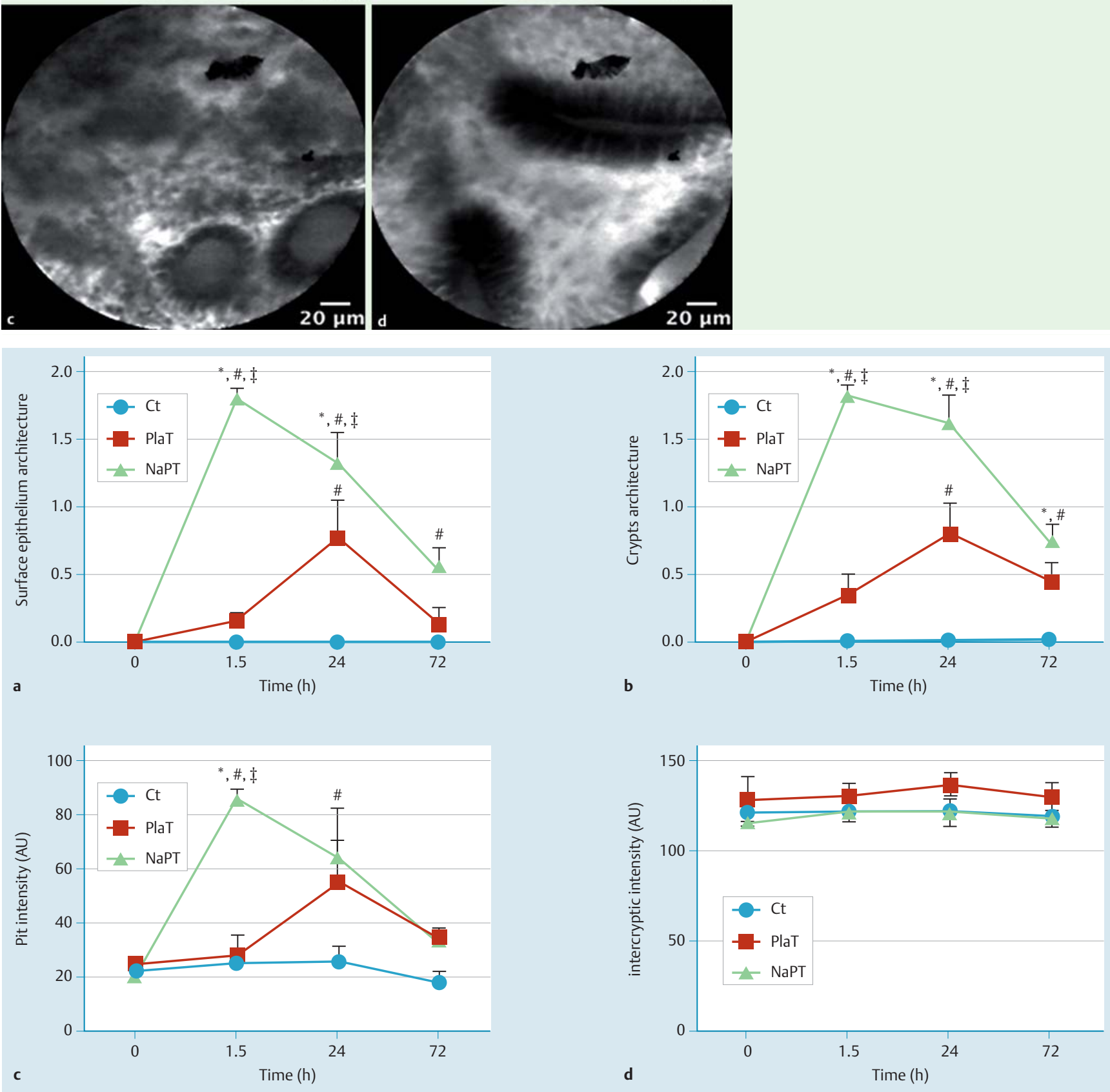

Fig. 5 Time course of endomicroscopic aspects at 1.5, 24, and 72 hours after the application of sodium phosphate tablets (NaPT) or placebo tablets (PlaT) in pigs. Control (Ct): unexposed mucosal area. a Semiquantitative lesion score of surface epithelium architecture. b Semiquantitative lesion score of crypts architecture. c Variations of fluorescein intensity in the crypt lumen $[(\mathrm{H} 1.5-\mathrm{H} 0) \times 100 / \mathrm{H} 0]$. d Variations of intercryptic intensity. Wilcoxon signed-rank test: ${ }^{*} P \leq 0.05$ vs. value at HO. Mann - Whitney test: ${ }^{*} P<0.05$ vs. value of $\mathrm{Ct}$ at identical time and $\ddagger P<0.05$ vs. value of PlaT at identical time. AU, arbitrary units. 
difficult and limited in humans [18]. In the present study, we developed an original approach for characterizing both the features of injury (macroscopic and microscopic) and the functional consequences of direct, drug-induced gastric mucosal toxicity. Our rationale for developing an experimental animal model to document drug-induced injuries in the gastric mucosa follows: (1) an animal model is ethical because serial endoscopic examinations over either a short or prolonged interval cannot be performed in humans; (2) the model allows the development of lesions to be monitored over a long period of time; and (3) large submucosal dissections can be done. The pig was chosen for its suitability as a model in terms of gastric anatomy, gastric size, and physiology, which are considered to be close to those of humans [19]. Furthermore, in this model, tools similar to those used in humans can be used for the morphologic and functional assessment of mucosal lesions.

We were able to place the tablets at the chosen sites and, more importantly, to keep them in place without difficulty for 1.5 hours. This was likely facilitated by the deep anesthesia of the animals, which helped to inhibit gastric wall motility. In these conditions of prolonged tablet application, we anticipated the induction of maximal injury to the exposed area.

Based on the macroscopic appearances, our results show the presence of mucosal lesions after NaPT application. These observations confirm previous findings in humans, especially concerning fundic injuries and injuries in the colon $[11,12,14]$. Although nonphysiologic and rather drastic experimental conditions were used, with direct and prolonged application of tablets onto a "dry" area of mucosa, we did not observe any grade 3 lesions. At 24 hours after the initial procedure, grade 1 and grade 2 lesions were observed in 3 of the 9 animals (33\%), whereas there were no lesions in the other 6 animals (66\%). In addition, 72 hours after the procedure, no lesion could be identified on the previously exposed gastric area. Thus, our design additionally documents the time course of injuries and their spontaneous reversibility. Observed injuries were reversible in less than 72 hours, which appears to be consistent with healing of the gastric mucosa following superficial lesions [20].

The pCLE analysis showed that microscopic architectural disorganization occurred early, reflected by variation in fluorescein intensity in the crypt lumen as early as 1.5 hours after the application. The placebo tablet did not induce any significant changes in any of the parameters studied. By contrast, 24 hours after the application of PlaT and NaPT, significant changes were observed for all parameters except intercryptic intensity. However, the changes were smaller with PlaT than with NaPT. Overall, this finding suggests that early macroscopic mucosal changes could be due to active compound (see below), whereas later effects (24 hours) might involve mechanical as well as excipient components ( $\nabla$ Fig.5). In fact, despite the observed pCLE changes, the functional consequences were probably minimal because (1) there was no change in mucosal permeability assessed with the Ussing chamber and (2) no morphologic change in mucosal thickness was noted.

The mechanisms and components involved in the occurrence of such lesions remain unknown. They are likely related to sodium monophosphate because similar observations have been reported with other compounds containing sodium phosphate $[11,12$, $14]$.

Despite the fact that this well-characterized animal model included a time course analysis, our study has some limitations. First, as always when an animal model is used, the data may not be considered to reflect human conditions strictly. In addition, the anesthetic conditions may represent a potential bias because the animals were maintained in one position, so that gastric motility was inhibited and changes in mucosal defense mechanisms or in gastric mucosal blood flow could not be formally excluded. However, despite its nonphysiologic character, this condition does allow the creation of maximal direct toxic effects on the mucosa. Finally, although endoscopic sessions were repeated in the animals, the relatively long sampling time period (48 hours between the 24-and 72-hour sessions) does not allow a precise definition of the time taken for complete mucosal healing.

Although no severe lesions were observed in this study, the methods presented here could be valuable for gaining pathophysiologic insight into the evolution of severe digestive mucosal lesions. For instance, the model could be used to test the potential additional effects of corticosteroid therapies, especially in combination with other drugs known to induce gastric injury, such as nonsteroidal anti-inflammatory drugs and aspirin or platelet inhibitors. Likewise, from a mechanistic point of view, it could be interesting to use such an experimental model to test for the effects of simultaneously administered gastroprotective agents.

In conclusion, this study documents, in particularly severe experimental conditions, the macroscopic and microscopic acute gastric injuries induced by NaPT, which are commonly used for colonoscopic bowel preparation in humans. It illustrates both the superficial nature and spontaneous reversibility of the lesions, with healing occurring in less than 72 hours. Although such experimental conditions are never observed in clinical practice, and no increased risk factor associated with any drug has been reported in these conditions, the results indicate that further studies are needed to document the potential consequences of gastric toxicity in patients with increased risk factors, such as those with a history of gastric ulcers or gastric hemorrhage.

\section{Financial Support}

$\nabla$

\section{Declaration of funding interests}

This study was funded in part by Mayoly Spindler (experimental costs only) and by INSERM.

Competing interests: Emmanuel Coron has served as a speaker for Mayoly Spindler and Pentax and as a consultant for Mauna Kea Technologies. Stanislas Bruley des Varannes has served as a speaker for Mayoly Spindler, is a consultant for Alfa Wassermann and Given Imaging, and has received research funding from Mayoly Spindler.

\section{References}

1 Millan MS, Gross P, Manilich E et al. Adenoma detection rate: the real indicator of quality in colonoscopy. Dis Colon Rectum 2008; 51: $1217-1220$

2 Petersen BT. Quality measures and credentialing in gastrointestinal endoscopy. Curr Opin Gastroenterol 2010; 26: 459-465

3 Rex DK. Colonoscopic withdrawal technique is associated with adenoma miss rates. Gastrointest Endosc 2000; 51: $33-36$

4 Sanchez W, Harewood GC, Petersen BT. Evaluation of polyp detection in relation to procedure time of screening or surveillance colonoscopy. Am J Gastroenterol 2004; 99: 1941 - 1945

5 Segnan N, Patnick S, von Karsa $L$ et al. European guidelines for quality assurance in colorectal cancer screening and diagnosis. Luxembourg: Publications Office of the European Union; 2010

6 Wexner SD, Beck DE, Baron TH et al. A consensus document on bowel preparation before colonoscopy: prepared by a task force from the American Society of Colon and Rectal Surgeons (ASCRS), the American 
Society for Gastrointestinal Endoscopy (ASGE), and the Society of American Gastrointestinal and Endoscopic Surgeons (SAGES). Gastrointest Endosc 2006; 63: 894-909

7 Chilton AP, O'Sullivan M, Cox MA et al. A blinded randomized comparison of a novel, low-dose, triple regimen with Fleet phospho-soda: a study of colon cleanliness, speed and success of colonoscopy. Endoscopy 2000; 32: 37-41

8 Kambe H, Yamaji Y, Sugimoto $T$ et al. A randomized controlled trial of sodium phosphate tablets and polyethylene glycol solution for polyp detection. J Dig Dis 2012; 13: 374-380

9 Hassan C, Bretthauer M, Kaminski MF et al. Bowel preparation for colonoscopy: European Society of Gastrointestinal Endoscopy (ESGE) guideline. Endoscopy 2013; 45: 142 - 150

10 Sacher-Huvelin S, Coron E, Gaudric M et al. Colon capsule endoscopy vs. colonoscopy in patients at average or increased risk of colorectal cancer. Aliment Pharmacol Ther 2010; 32: 1145-1153

11 Nam SY, Choi IJ, Park KW et al. Risk of hemorrhagic gastropathy associated with colonoscopy bowel preparation using oral sodium phosphate solution. Endoscopy 2010; 42: 109-113

12 Coton T, Luciano L, Marcelko A. Colic lesions induced by Colokit preparation. Clin Res Hepatol Gastroenterol 2011; 35: 509-510

13 Zwas FR, Cirillo NW, EI-Serag HB et al. Colonic mucosal abnormalities associated with oral sodium phosphate solution. Gastrointest Endosc 1996; 43: $463-466$
14 Schir E, Barbouy-des-Courières S, Plottin F et al. [Colokit preparation (sodium phosphate) induced erosive gastritis]. Therapie 2012; 67: $477-479$

15 Hagège $H$, Ponchon $T$, Laugier $R$ et al. Etude des conditions d'utilisation et de la sécurité d'emploi d'un phosphate de sodium sous forme de comprimés, en France. Les Journées Francophones d'Hépato-gastroentérologie et d'Oncologie Digestive 2013: 173

16 Coskun A, Uzunkoy A, Duzgun SA et al. Experimental sodium phosphate and polyethylene glycol induce colonic tissue damage and oxidative stress. Br J Surg 2001; 88: 85-89

$17 \mathrm{Li} \mathrm{CQ}$ Xie XJ, Yu T et al. Classification of inflammation activity in ulcerative colitis by confocal laser endomicroscopy. Am J Gastroenterol 2010; 105: $1391-1396$

18 Fiorucci S, Santucci L, Gresele P et al. Gastrointestinal safety of NO-aspirin (NCX-4016) in healthy human volunteers: a proof of concept endoscopic study. Gastroenterology 2003; 124: 600-607

19 Batzri S, Harmon JW, Dubois A et al. A new in vivo method for repeatedly studying gastric acid secretion and other secretory parameters in awake guinea pig. J Surg Res 1987; 43: 398 - 406

20 Yeomans ND, Skeljo MV. Repair and healing of established gastric mucosal injury. J Clin Gastroenterol 1991; 13: 37-41 